\title{
Identification of circulating microRNA signatures for upper tract urothelial carcinoma detection
}

\author{
JUN TAO $^{1 *}$, XIAO YANG $^{1 *}$, PENGCHAO LI $^{1 *}$, JIFU WEI $^{2}$, XIAHENG DENG $^{1}$, YIDONG CHENG $^{1}$, \\ CHAO QIN ${ }^{1}$, XIAOBING JU ${ }^{1}$, XIAOXIN MENG ${ }^{1}$, JIE LI $^{1}$, MIN GU ${ }^{1}$, QIANG LU ${ }^{1}$ and CHANGJUN YIN ${ }^{1}$ \\ ${ }^{1}$ Department of Urology; ${ }^{2}$ Clinical Research Centre, \\ The First Affiliated Hospital of Nanjing Medical University, Nanjing, Jiangsu 210029, P.R. China
}

Received November 13, 2014; Accepted August 6, 2015

DOI: $10.3892 / \mathrm{mmr} .2015 .4257$

\begin{abstract}
The involvement of circulating microRNAs (miRNAs) in cancer and their potential as diagnostic and prognostic biomarkers are becoming increasingly known. However, the significance of circulating miRNAs in upper tract urothelial carcinoma (UTUC) has remained to be investigated. The present study performed a genome-wide serum miRNA analysis using a deep sequencing platform for initial screening. Subsequently, serum samples of 46 UTUC patients and 30 cancer-free individuals with hematuria were subjected to a quantitative reverse-transcription polymerase chain reaction analysis. The expression of thirteen miRNAs (miR-664a-3p, miR-423-5p, miR-431-5p, miR-191-5p, miR-92a-3p, miR-22-3p, miR-26a-5p, miR-33b-3p, miR-16-5p, let-7a-5p, let-7b-5p, let-7f-5p and let-7c) was significantly different in serum from UTUC patients compared with that in control samples. Receiver operator characteristic analysis showed that 10 miRNAs (miR-664a-3p, miR-431-5p, miR-423-5p, miR-191-5p, miR-33b-3p, miR-26a-5p, miR-22-3p, miR-16-5p, let-7b-5p and let-7c) had the potential to distinguish individuals with UTUC from the controls (areas under the curve $>0.8$ ). The present study provided the first evidence for the potential use of circulating miRNAs as biomarkers for UTUC diagnosis, which remains to be verified by further studies.
\end{abstract}

\section{Introduction}

Upper tract urothelial carcinoma (UTUC) is an uncommon, clinically heterogeneous and potentially fatal disease. UTUC

Correspondence to: Dr Min Gu or Dr Qiang Lu, Department of Urology, The First Affiliated Hospital of Nanjing Medical University, 300 Guangzhou Road, Nanjing, Jiangsu 210029, P.R. China

E-mail: lancetgu@aliyun.com

E-mail: doctorlvqiang@sina.com

${ }^{*}$ Contributed equally

Key words: microRNA, upper tract urothelial carcinoma, serum, circulating, sequencing accounts for only $5-10 \%$ of urothelial carcinomas $(1,2)$ and $60 \%$ of UTUCs are invasive at diagnosis compared with only $15 \%$ for bladder tumors $(1,3)$. The established early diagnostic methods for UTUC, including multi-detector computed tomographic urography, magnetic resonance imaging, urinary cytology, cystoscopy and ureteroscopy, are either invasive or lack sensitivity (3). Thus, the majority of UTUC cases are diagnosed at an advanced stage, resulting in poor prognoses. Results from studies on bladder cancer, which is more common and more thoroughly studied, are often extrapolated to UTUC (4); however, molecular markers for the detection of UTUC are largely absent. Individuals with low-risk UTUC can partially benefit from conservative therapy $(3,5)$, but radical nephroureterectomy is still considered the gold standard for the treatment of localized UTUC $(3,6)$. Therefore, correct early diagnosis is critical for designing appropriate treatment schedules to increase survival and decrease morbidity. Early biomarkers that can complement and improve current strategies for UTUC detection should be identified.

MicroRNAs (miRNAs/miRs) are a class of 17-27 nucleotide (nt) single-stranded RNA molecules that can regulate gene expression by repressing translation or cleaving RNA transcripts in a sequence-specific manner. The biogenesis of miRNA has been comprehensively reported in numerous reviews $(7,8)$, and the ability of miRNAs to inhibit the translation of oncogenes and tumor suppressors suggests their involvement in carcinogenesis $(9,10)$. miRNAs present in human blood samples are known as circulating miRNAs. Circulating miRNAs have been recently demonstrated to be present in a stable and reproducible state (11). Serum and plasma samples are easily acquired in a relatively non-invasive manner, and isolated miRNAs can be readily detected by reverse-transcription quantitative polymerase chain reaction (RT-qPCR), which is a widely used clinical and laboratory technique. The unique expression profile of serum or plasma miRNA has been used as a fingerprint for various malignancies, including carcinomas of the urinary tract $(12,13)$. Extracellular miRNAs, either embedded in microvesicles or protected by RNA-binding proteins/lipids, can provide diagnostic, prognostic or even therapeutic targets for UTUC.

However, in spite of the aberrant expression of miRNAs being implicated in the clinical prognosis of human UTUC (14), the global circulating miRNA patterns in UTUC patients have 
not been studied, to the best of our knowledge. Circulating miRNAs are promising candidates for non-invasive cancer testing due to the stability and tissue specificity of miRNAs in the blood. Circulating miRNAs have been recently reported as diagnostic markers for various cancer types (15-18). The present study used the deep sequencing platform Illumina HiSeq $^{\mathrm{TM}} 2000$, followed by RT-qPCR, to systematically and extensively identify circulating miRNAs as a novel class of potent non-invasive biomarkers for UTUC.

\section{Materials and methods}

Ethics statement. The present study was approved by the Institutional Review Board of the First Affiliated Hospital of Nanjing Medical University (Nanjing, China). Written informed consent was obtained from all participants involved in this study.

Study population, sample collection and serum RNA isolation. The present study collected serum samples from 58 UTUC patients (mean age, $68.0 \pm 11.6$ years) undergoing radical nephroureterectomy at the First Affiliated Hospital of Nanjing Medical University (Nanjing, China), from 2007 to 2013. A total of 42 matched cancer-free controls (mean age, $67.9 \pm 9.0$ years) were also included. These controls were patients with hematuria who were further diagnosed with urinary tract infection, benign prostate hyperplasia and urinary tract calculi at the First Affiliated Hospital of Nanjing Medical University (Nanjing, China). Clinical and pathological characteristics, including age, gender, family history of cancer, stage and grade, were recorded and are summarized in Table I. The diagnosis of UTUC was established according to the results of histopathological analysis. Tumors were graded and classified according to the WHO (2004) and TNM classifications of the International Union Against Cancer (2009) $(19,20)$. Blood samples of all patients were drawn within $24 \mathrm{~h}$ after admission. The coagulated blood samples were collected in tubes containing a separating gel and clot activator and centrifuged at $1,500 \mathrm{x} \mathrm{g}$ for $15 \mathrm{~min}$ at $4^{\circ} \mathrm{C}$. The supernatant was centrifuged at $1,500 \mathrm{x}$ g for $15 \mathrm{~min}$ to precipitate cell debris and stored at $-80^{\circ} \mathrm{C}$ until use. Total RNA of 12 UTUC patients and 12 controls was isolated from $200-\mu 1$ serum samples using an miRNeasy Serum/Plasma kit (Qiagen, Hilden, Germany) according to the manufacturer's instructions.

HiSeq 2000 sequencing and bioinformatics analysis. Small RNAs of 16-30 nt in length were first isolated from the total RNA by size fractionation in a $15 \%$ Tris/borate/EDTA urea polyacrylamide gel, and these small RNAs were ligated to an activated $5^{\prime}$ adaptor. A 3' adaptor was then ligated to the small RNA-5' adaptor, and RT using a RT primer was performed to create cDNA constructs. The RT reaction was performed using primers that were complementary to the two adaptors. The amplified cDNA constructs were purified and sequenced using an Illumina HiSeq 2000 platform (Illumina, Inc., San Diego, CA, USA) with the following parameters: The adaptor molecule and empty sequence were directly trimmed, and low-quality tags were filtered. Only reads $18-32$ bp-reads were selected. All sequences that matched transfer RNA, ribosomal RNA and DNA repeats were filtered. Thus, two types of alignment were generated: Reads uniquely mapped to known miRNAs and reads generating other unknown small RNAs. The expression of miRNAs was calculated, and DEGseq was used to differentially screen the miRNAs between the cases and controls by the criterion of $\mathrm{P}<0.001$. All of the abovementioned procedures were performed by Shanghai Majorbio Bio-pharm Technology (Shanghai, China).

miRNA quantification by $R T-q P C R$. A two-phase case-control study was designed to identify serum miRNAs as potential markers for UTUC. In the training set, miRNAs were measured in serum samples from 12 UTUC patients and 12 controls. In the validation set, miRNAs were measured in serum samples from 46 UTUC patients and 30 controls. miRNAs were prepared using an All-in-One ${ }^{\mathrm{TM}}$ miRNA qRT-PCR Detection kit (cat. no. AOMD-Q060; GeneCopoeia-FulenGen, Guangzhou, China) according to the manufacturer's instructions. Extracted RNA was reverse-transcribed in the presence of poly-A polymerase with an oligo-dT adaptor. A SYBR green qPCR assay was performed with an Applied Biosystems Step One Plus System (Applied Biosystems, Foster City, USA) with forward primer for the mature miRNA sequence and a universal adaptor reverse primer. The respective validated miRNA-specific forward primer (GeneCopoeia-FulenGen) was used for the qPCR assay. Amplification was performed under the following conditions: $95^{\circ} \mathrm{C}$ for $10 \mathrm{~min}$, followed by 45 cycles of $95^{\circ} \mathrm{C}$ for $15 \mathrm{sec}, 60^{\circ} \mathrm{C}$ for $30 \mathrm{sec}$ and $72^{\circ} \mathrm{C}$ for $40 \mathrm{sec}$. The expression levels of miRNA were normalized to RNU6-2 as described previously (21). All reactions, including controls with no template RNA, were performed in triplicate. The relative expression of miRNA compared with RNU6-2 was calculated using the $2^{-\Delta \mathrm{Ct}}$ method.

Statistical analysis. Student's $t$-test or the $\chi^{2}$ test were used to determine the differences in the selected demographic variables between the cases and controls. The relative expression of miRNA was analyzed using the $2^{-\Delta \Delta \mathrm{Ct}}$ method. The Mann-Whitney $U$ test was used to compare the expression of serum miRNAs between the different groups. R software (version 3.0.1; MathSoft, Seattle, WA, USA) was used to run unsupervised clustering analysis and principal component analysis (PCA). Sensitivity, specificity and area under the curve (AUC) for serum miRNA levels were determined using receiver operator characteristic (ROC) analysis. All tests were two-sided, and $\mathrm{P}<0.05(95 \% \mathrm{CI})$ was considered to indicate a statistically significant difference. Statistical analysis was performed using SPSS 13.0 software (SPSS, Inc., Chicago, IL, USA).

\section{Results}

Characteristics of the study population. The frequency distributions of the selected characteristics of the cases and controls are shown in Table I. No significant differences in age, gender, tobacco smoking and alcohol consumption were identified between the UTUC patients and controls ( $\mathrm{P}>0.05)$. The family history of malignancies was similar between the cases and controls. The clinicopathological characteristics are presented in Table I. 

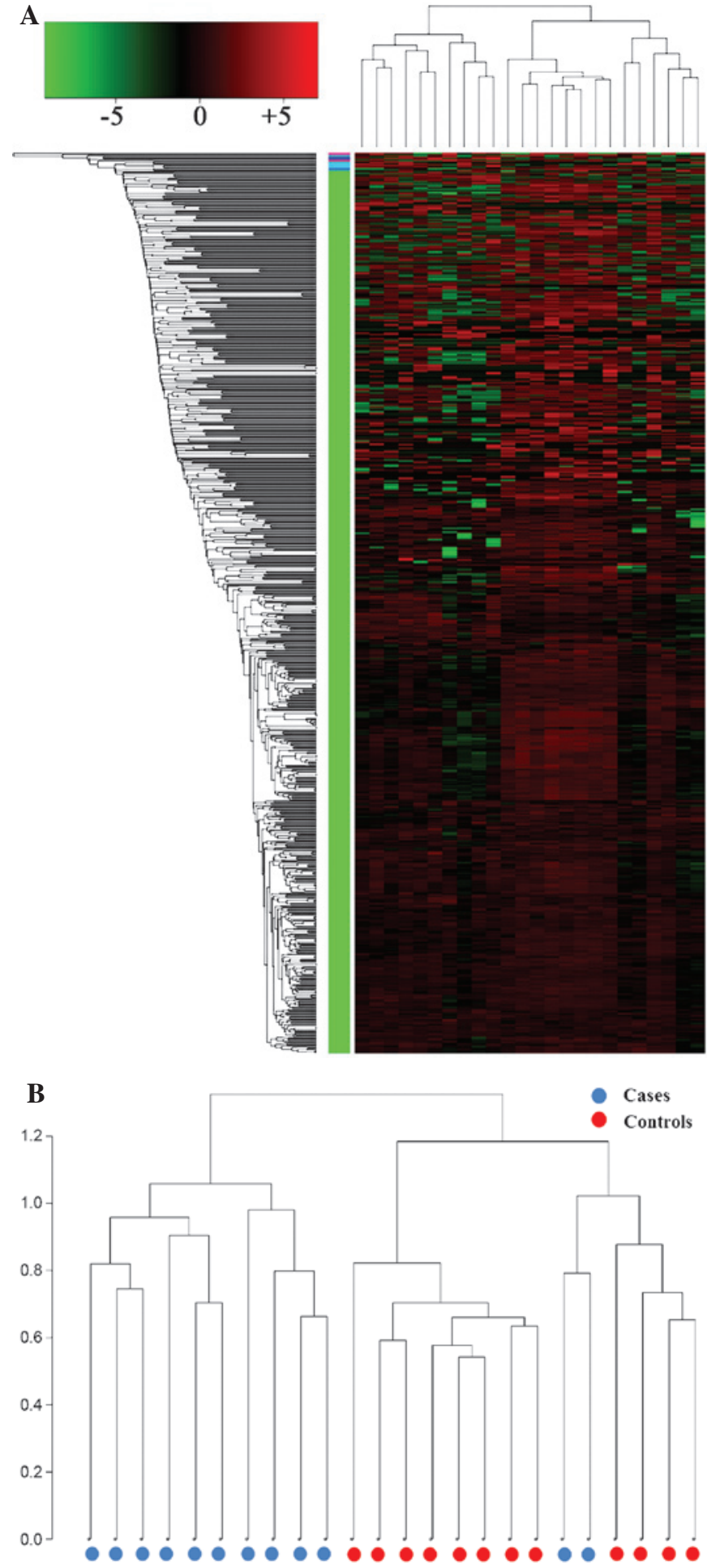

Figure 1. (A) Heat map and (B) cluster dendrogram of differentially expressed microRNAs.

Unsupervised clustering analysis and PCA. The differential expression of miRNAs between the UTUC and control serum samples was analyzed by performing unsupervised clustering that was blinded to the clinical annotations. The dendrogram generated by cluster analysis showed a clear distinction between the UTUC and control samples based on the serum miRNA profile (Fig. 1).

PCA was used to classify 24 samples (12 UTUC and 12 control samples) based on the expression profile of

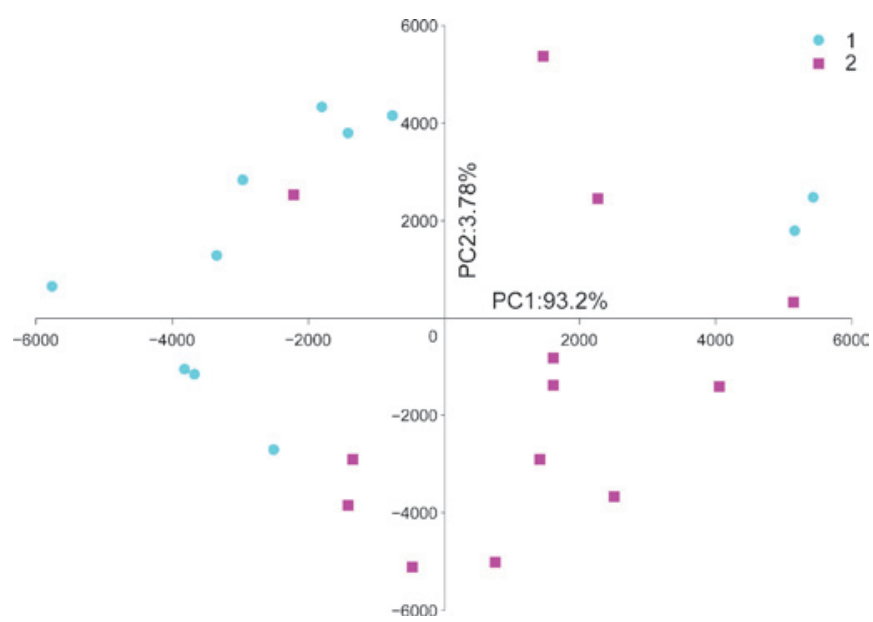

Figure 2. Unsupervised classification by PCA of cases and control samples. PCA was used to classify 24 samples (12 cases and 12 controls) based on the expression profile of 711 microRNAs expressed in all samples. PCA, principal component analysis; 1 , controls; 2 , cases.

711 miRNAs expressed in all samples. The PCA plot illustrates the global characteristics of these samples (Fig. 2). Despite heterogeneity within the two groups, the first principal component (PC1) was able to differentiate the UTUC samples from the control samples, and the difference between the two groups was $>95 \%$.

RT-qPCR analysis of serum miRNAs in UTUC. To validate the putative markers identified from the profiling results, the present study confirmed the concentrations of 21 candidate miRNAs, which were selected following HiSeq 2000 sequencing, with a SYBR green-based RT-qPCR assay. The criteria for selecting miRNA for RT-qPCR analysis were as follows: i) Counts of miRNAs $>100$ in all sequenced samples; ii) fold change $>2$ or $<0.5$ and $\mathrm{P}<0.001$; iii) the absolute value of the coefficient in PC1 $>500$; and iv) miRNAs sub-categorized by the unsupervised clustering method. Consequently, 21 miRNAs that met the inclusion criteria were selected for further analysis. The results are summarized in Table II.

Within the validation set, the miRNAs of individual serum samples from 46 UTUC patients and 30 controls were measured, and only miRNAs with a $\mathrm{Ct}$ value $<35$ in either the UTUC or control groups were selected for further analysis. Furthermore, miRNAs with a detection rate of $<80 \%$ and $\mathrm{P}>0.05$ were excluded from further analysis. These criteria were used to generate a list of 13 miRNAs (miR-664a-3p, miR-423-5p, miR-431-5p, miR-191-5p, miR-92a-3p, miR-22-3p, miR-26a-5p, miR-33b-3p, miR-16-5p, let-7a-5p, let-7b-5p, let-7f-5p and let-7c) that showed different miRNA patterns between the UTUC patients and controls (Fig. 3).

ROC curve analysis. The ROC curves that were constructed to compare the relative concentrations of the 13 miRNAs between the UTUC patients and controls yielded the following AUCs: miR-664a-3p, 0.998, 95\% CI (0.992-1.000); miR-423-5p, $0.802,95 \%$ CI (0.702-0.902); miR-431-5p, 0.933, 95\% CI (0.879-0.988); miR-191-5p, 0.814, 95\% CI (0.712-0.917); miR-92a-3p, 0.675, 95\% CI (0.552-0.797); miR-22-3p, 0.836, 95\% CI (0.741-0.931); miR-26a-5p, 0.891, 95\% CI (0.861-0.962); 
Table I. Demographic and clinical features of patients with upper tract urothelial carcinoma and controls in the training and validation sets.

\begin{tabular}{|c|c|c|c|c|c|}
\hline \multirow[b]{2}{*}{ Variable } & \multicolumn{2}{|c|}{ Cases $(\mathrm{n}=58)$} & \multicolumn{2}{|c|}{ Controls $(n=42)$} & \multirow[b]{2}{*}{ P-value ${ }^{a}$} \\
\hline & $\mathrm{n}$ & $\%$ & $\mathrm{n}$ & $\%$ & \\
\hline \multicolumn{6}{|l|}{ Age (years) } \\
\hline$<68$ & 28 & 48.3 & 22 & 52.4 & \multirow[t]{2}{*}{0.685} \\
\hline$\geq 68$ & 30 & 51.7 & 20 & 47.6 & \\
\hline \multicolumn{6}{|l|}{ Gender } \\
\hline Male & 41 & 29.3 & 30 & 71.4 & \multirow[t]{2}{*}{0.936} \\
\hline Female & 17 & 70.7 & 12 & 28.6 & \\
\hline \multicolumn{6}{|c|}{ Tobacco smoking status } \\
\hline Never & 32 & 55.2 & 26 & 61.9 & \multirow[t]{2}{*}{0.501} \\
\hline Positive $^{\mathrm{b}}$ & 26 & 44.8 & 16 & 38.1 & \\
\hline \multicolumn{6}{|c|}{ Alcohol consumption status } \\
\hline Never & 40 & 82.8 & 31 & 73.8 & \multirow[t]{2}{*}{0.278} \\
\hline Positive $^{\mathrm{b}}$ & 18 & 17.2 & 11 & 26.2 & \\
\hline \multicolumn{6}{|c|}{ Family history of cancer } \\
\hline No & 42 & 72.4 & 37 & 88.1 & \multirow[t]{2}{*}{0.057} \\
\hline Yes & 16 & 27.6 & 5 & 11.9 & \\
\hline \multicolumn{6}{|l|}{ Tumor stage } \\
\hline I & 21 & 36.2 & & & \\
\hline II & 14 & 24.1 & & & \\
\hline III & 20 & 34.5 & & & \\
\hline IV & 3 & 5.2 & & & \\
\hline \multicolumn{6}{|l|}{ Tumor grade } \\
\hline PUNLMP & 1 & 1.7 & & & \\
\hline Low-grade & 17 & 29.3 & & & \\
\hline High-grade & 40 & 69.0 & & & \\
\hline
\end{tabular}

${ }^{\mathrm{a}} \mathrm{P}=0.934$ according to Student's $t$-test for age distribution between the cases and controls; two-sided $\chi^{2}$ test for other selected variables between the cases and controls. 'Includes either history of use or present use. PUNLMP, papillary urothelial neoplasia of low malignant potential.

miR-33b-3p, 0.879, 95\% CI (0.801-0.958); miR-16-5p, 0.821, 95\% CI (0.715-0.917); let-7a-5p, 0.725, 95\% CI (0.605-0.845); let-7b-5p, 0.856, 95\% CI (0.762-0.949); let-7f-5p, 0.642, 95\% CI (0.516-0.768); and let-7c, 0.905, 95\% CI (0.836-0.974). These results suggested the potential of miRNAs for discriminating patients with UTUC from control subjects (Fig. 4).

Association with clinical characteristics. To demonstrate the increase in serum miRNA expression during UTUC development, UTUC cases were further stratified according to tumor stage and grade. The patients were divided into two groups (muscle invasive and non-muscle invasive) according to pathological stage, and three groups according to pathological grade. No statistically significant differences were observed among the miRNAs; miR-664a-3p, miR-431-5p, miR-423-5p, miR-191-5p, miR-92a-3p, miR-16-5p and let-7b-5p were all upregulated in the muscle-invasive group. Similarly, no statistically significant difference was identified when members of the UTUC group were stratified according to tumor grade (data not shown).

\section{Discussion}

The present study evaluated the use of circulating miRNA levels as diagnostic and prognostic factors for UTUC in order to improve currently used diagnostic tools. The results showed that UTUC was associated with a significant increase in the serum levels of 13 circulating miRNAs, namely miR-664a-3p, miR-423-5p, miR-431-5p, miR-191-5p, miR-92a-3p, miR-22-3p, miR-26a-5p, miR-33b-3p, miR-16-5p, let-7a-5p, let-7b-5p, let-7f-5p and let-7c. Further ROC curve analysis showed that ten of the 13 miRNAs (miR-664a-3p, miR-431-5p, miR-423-5p, miR-191-5p, miR-33b-3p, miR-26a-5p, miR-22-3p, miR-16-5p, let-7b-5p and let-7c) were potentially suitable for distinguishing UTUC individuals from the controls (AUC $>0.8$ ).

Circulating miRNAs are present in body fluids and dysregulated miRNAs may be used as disease markers due to their small size, stability and pivotal involvement in the regulation of cell function (22). Tumor-derived miRNAs in serum or plasma are easily accessible and the detection technology is widely used in clinical settings. Therefore, circulating miRNAs may 
Table II. Differentially expressed miRNAs between serum of patients with upper tract urothelial carcinoma and controls.

\begin{tabular}{|c|c|c|c|c|}
\hline miRNA name & $\begin{array}{l}\text { Lowest count in } \\
\text { all samples }\end{array}$ & $\begin{array}{c}\text { Fold change (cases } \\
\text { vs. controls) }\end{array}$ & P-value & Predicted by \\
\hline hsa-let-7a-5p & 88495 & 2.08 & $<0.001$ & PCA \\
\hline hsa-let-7b-5p & 13358 & 3.18 & $<0.001$ & PCA \\
\hline hsa-let-7c & 65799 & 2.44 & $<0.001$ & PCA \\
\hline hsa-let-7f-5p & 85201 & 2.05 & $<0.001$ & PCA \\
\hline hsa-miR-16-5p & 477766 & 2.43 & $<0.001$ & PCA \\
\hline hsa-miR-183-5p & 7393 & 4.24 & $<0.001$ & PCA \\
\hline hsa-miR-191-5p & 53946 & 2.57 & $<0.001$ & PCA \\
\hline hsa-miR-22-3p & 64832 & 0.46 & $<0.001$ & PCA \\
\hline hsa-miR-25-3p & 86758 & 2.78 & $<0.001$ & PCA \\
\hline hsa-miR-26a-5p & 14980 & 2.33 & $<0.001$ & PCA \\
\hline hsa-miR-33b-3p & 6636 & 3.98 & $<0.001$ & CA \\
\hline hsa-miR-3609 & 127 & 12.68 & $<0.001$ & $\mathrm{CA}$ \\
\hline hsa-miR-370 & 106 & 0.39 & $<0.001$ & $\mathrm{CA}$ \\
\hline hsa-miR-423-5p & 9077 & 2.48 & $<0.001$ & PCA \\
\hline hsa-miR-431-5p & 169 & 0.23 & $<0.001$ & $\mathrm{CA}$ \\
\hline hsa-miR-451a & 183231 & 2.18 & $<0.001$ & PCA \\
\hline hsa-miR-4763-5p & 122 & 10.81 & $<0.001$ & $\mathrm{CA}$ \\
\hline hsa-miR-486-5p & 1537466 & 2.99 & $<0.001$ & PCA \\
\hline hsa-miR-655 & 148 & 5.18 & $<0.001$ & $\mathrm{CA}$ \\
\hline hsa-miR-664a-3p & 24258 & 8.12 & $<0.001$ & CA \\
\hline hsa-miR-92a-3p & 161639 & 0.46 & $<0.001$ & PCA \\
\hline
\end{tabular}

CA, cluster analysis; PCA, principal components analysis; hsa, Homo sapiens; miR/miRNA, microRNA.

Table III. Diagnostic values of 13 miRNAs with regard to detection of UTUC.

\begin{tabular}{|c|c|c|c|c|c|c|}
\hline miRNA name & AUC & $95 \% \mathrm{CI}$ & P-value & Cut-off value ${ }^{a}$ & Sensitivity & Specificity \\
\hline hsa-let-7a-5p & 0.725 & $0.605-0.845$ & 0.001 & 31.55 & 0.696 & 0.833 \\
\hline hsa-let-7b-5p & 0.856 & $0.762-0.949$ & $<0.001$ & 15.15 & 0.761 & 0.867 \\
\hline hsa-let-7c & 0.905 & $0.836-0.974$ & $<0.001$ & 1.53 & 0.739 & 0.967 \\
\hline hsa-let-7f-5p & 0.642 & $0.516-0.768$ & 0.037 & 7.06 & 0.674 & 0.700 \\
\hline hsa-miR-16-5p & 0.821 & $0.725-0.917$ & $<0.001$ & 290.05 & 0.739 & 0.867 \\
\hline hsa-miR-191-5p & 0.814 & $0.712-0.917$ & $<0.001$ & 12.92 & 0.848 & 0.800 \\
\hline hsa-miR-22-3p & 0.836 & $0.741-0.931$ & $<0.001$ & 21.40 & 0.870 & 0.800 \\
\hline hsa-miR-26a-5p & 0.891 & 0.821-0.962 & $<0.001$ & 33.17 & 0.804 & 0.883 \\
\hline hsa-miR-33b-3p & 0.879 & $0.801-0.958$ & $<0.001$ & 313.26 & 0.717 & 0.967 \\
\hline hsa-miR-423-5p & 0.802 & 0.702-0.902 & $<0.001$ & 30.01 & 0.674 & 0.867 \\
\hline hsa-miR-431-5p & 0.933 & 0.879-0.988 & $<0.001$ & 0.83 & 0.826 & 0.933 \\
\hline hsa-miR-664a-3p & 0.998 & 0.992-1.000 & $<0.001$ & 113.74 & 0.978 & 1.000 \\
\hline hsa-miR-92a-3p & 0.675 & $0.552-0.797$ & 0.010 & 338.44 & 0.587 & 0.867 \\
\hline
\end{tabular}

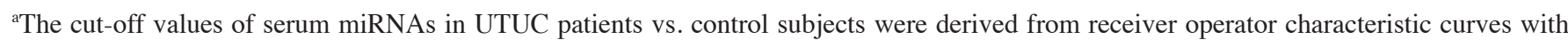
Youden's index. UTUC, upper tract urothelial carcinoma; hsa, Homo sapiens; miR/miRNA, microRNA; CI, confidence interval; AUC, area under curve.

emerge as potentially useful blood-based tools for monitoring human cancers, particularly at an early stage (23). In the present study, the deep sequencing platform Illumina HiSeq ${ }^{\mathrm{TM}} 2000$ was used to preliminarily characterize an index for estimating the abundance of circulating miRNAs from 12 UTUC patients and 12 controls as an initial screening stage, allowing for the determination of the differential expression of 21 miRNAs between UTUC patients and controls. Deep-sequencing 

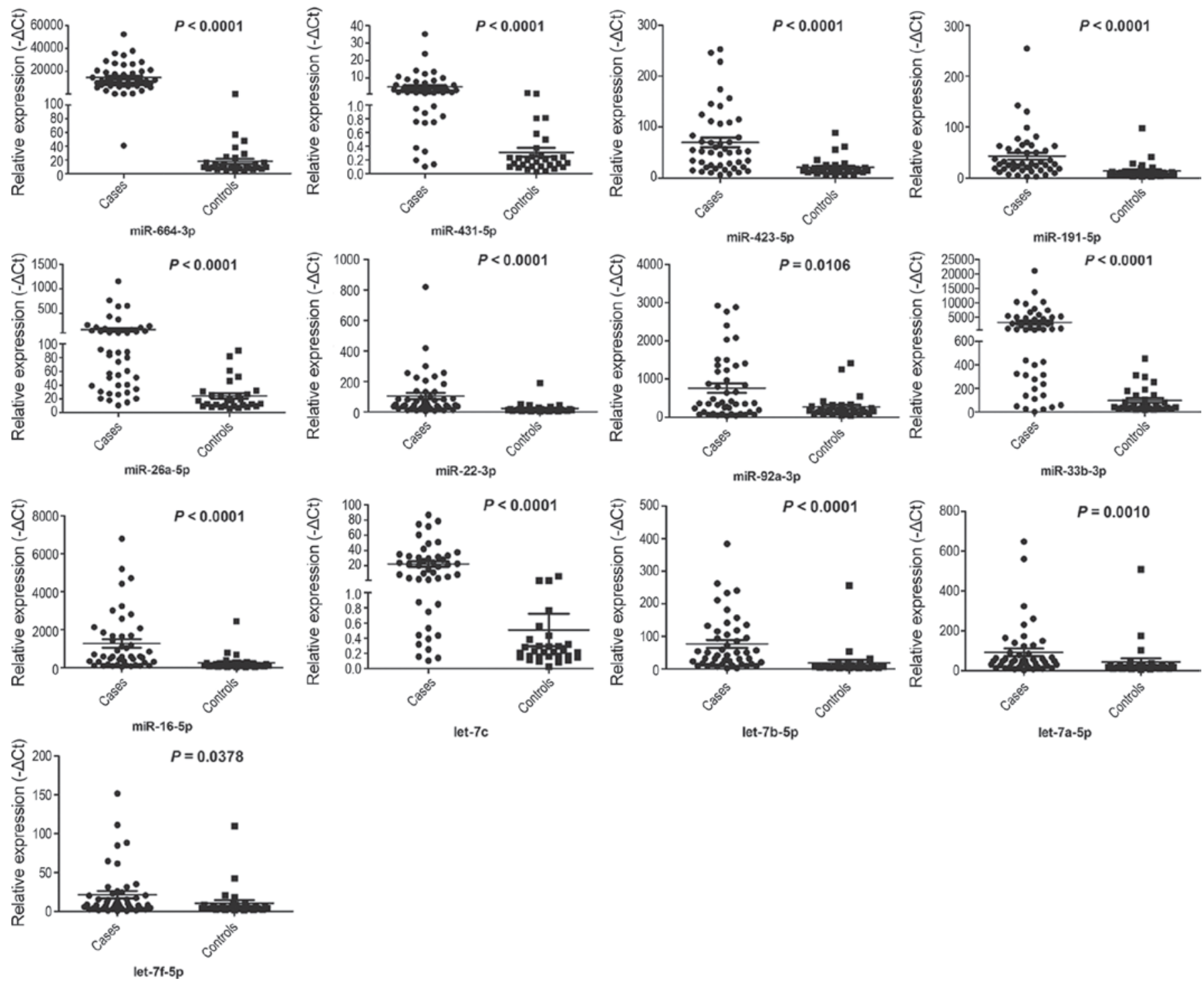

let-7c

let-7a-5p

Figure 3. Relative levels of 13 serum miRNAs in 46 upper tract urothelial carcinoma patients and 30 control individuals (in the validation sets; mean \pm standard deviation). These levels were determined by reverse transcription quantitative polymerase chain reaction analysis. Each point represents the mean result for triplicate samples. miR/miRNA, microRNA; Ct, cycle threshold.

approaches for miRNA analysis are becoming routine, and the number of studies targeting miRNAs as potential diagnostic biomarkers for diseases is increasing (24). The major benefits of next-generation sequencing for miRNA profiling include detection of novel as well as known miRNAs and precise identification of miRNA sequences (25). Deep sequencing technology is an effective high-throughput tool for initial serum miRNA screening with high accuracy and sensitivity. However, the sequencing results should be validated by RT-qPCR using a large number of individual serum samples, because the sequencing results were obtained from pooled serum samples and may include inaccurate information caused by individual variations. After the initial stage of sequencing circulating miRNA-based cancer biomarkers, the present study used RT-qPCR to assess the relative miRNA expression. Subsequently, a list of 13 miRNAs with differential expression between the UTUC cases $(n=46)$ and controls $(n=30)$ was generated. The present study retrospectively assessed the diagnostic capacity of the 13 miRNAs and identified 10 miRNAs that exhibited significant differences (AUC >80\%) between the UTUC cases and the controls, therefore being suitable for distinguishing between the two groups. Circulating miRNAs identified by deep sequencing technology were recently considered as fingerprints for the diagnosis of other types of solid tumor, including gastric cancer (15), papillary thyroid carcinoma (16), hepatocarcinoma (17) and malignant astrocytomas (18). Furthermore, miRNAs isolated from urine or blood in bladder cancer patients were also reported to show potential as non-invasive biomarkers for diagnosis and prognosis (26-28).

Among the 10 serum miRNAs identified in the present study, several have already been reported to function in detecting disease, creating prognosis or monitoring treatment response. The expression of circulating miRNA precursor let-7b was reported to be diverse among various cancer types. Let-7b was found to be upregulated in breast tumors (29) and downregulated in ovarian cancer (30). The same trend was found for the expression of circulating let-7c, which was increased in esophageal cancer (31) but decreased in nasopharyngeal carcinoma patients with poor prognosis (32). 

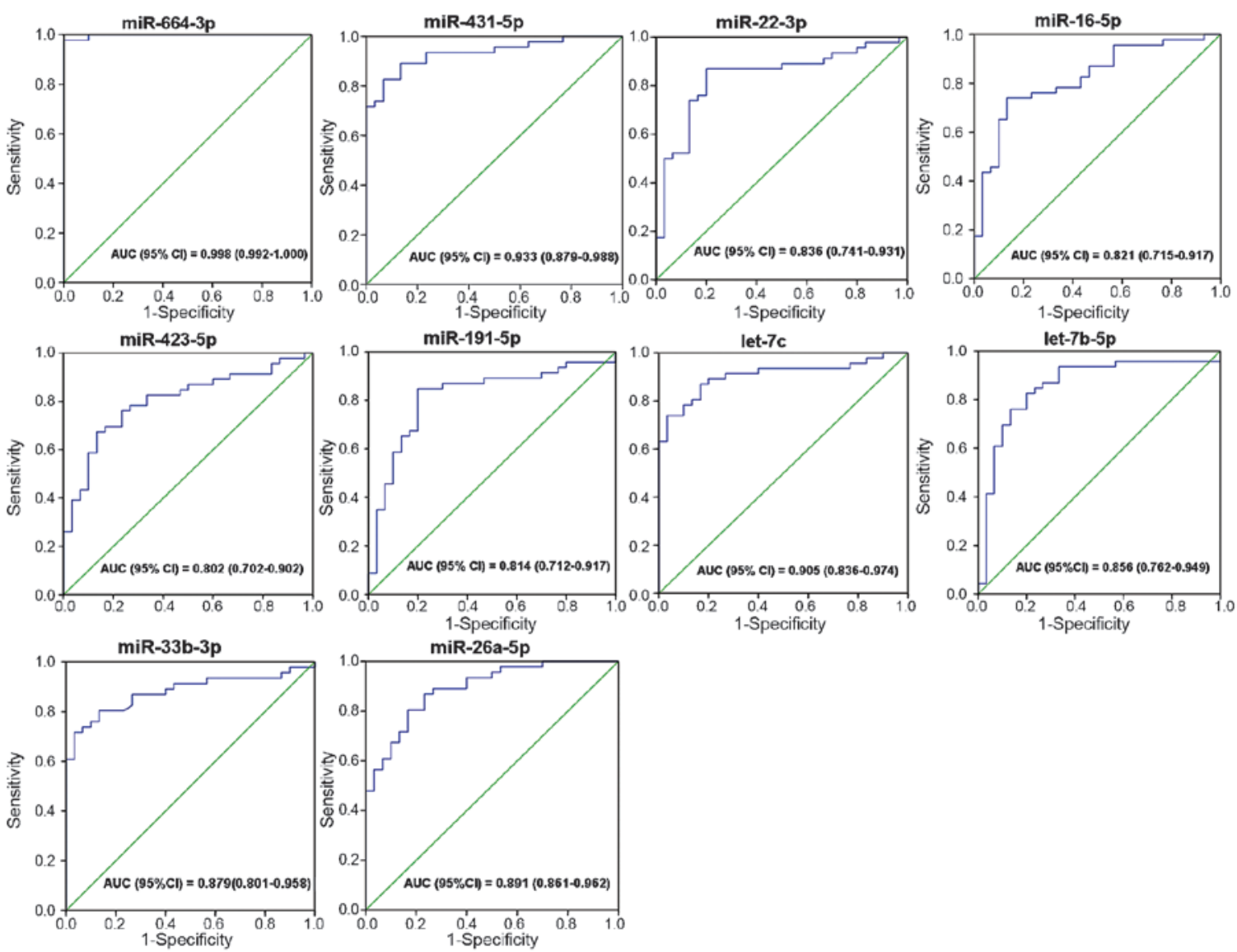

Figure 4. Receiver operator characteristic curves reflecting the ability of the serum levels of 10 individual miRNAs to distinguish the upper tract urothelial carcinoma cases $(n=46)$ from the controls $(n=30)(A U C>80 \%)$. miR/miRNA, microRNA; AUC, area under curve; CI, confidence interval.

Circulating let-7b and let-7c expression is cancer-specific. Let-7b is highly expressed in platelets (33), which may obscure the diagnostic value of measuring let-7b expression levels in serum. Circulating miR-22 was first reported in esophageal squamous cell carcinoma (ESCC) and serves as a non-invasive biomarker for ESCC diagnosis (34). High expression of miR-22 can explain the lack of response in pemetrexed-treated non-small cell lung cancer (NSCLC) patients. However, in primary plasma cell leukemia, miR-22 is associated with a positive clinical outcome (35). One study suggested that circulating miR-431 can contribute to the detection of colorectal cancer (36). The present study found that serum let-7b, miR-22, and miR-431 were suitable for distinguishing UTUC cases from the controls with AUCs of 85.6, 83.6 and 93.3\%, respectively. As mentioned above, circulating let-7b, miR-22 and miR-431 have been linked to the pathogenesis of other cancer types, indicating the value of these three miRNAs in detecting cancer, including UTUC.

Circulating miR-16 and miR-191 have been reported as reference genes in colorectal adenocarcinoma (37). However, miR-16 and miR-191 are associated with the pathogenesis of other cancer types, including NSCLC (38) and lymphoma (39). Of note, several serum miRNAs identified in the present study are also correlated with urinary cancers. For instance, serum miR-16 and miR-26a allow for the discrimination of prostate cancer and benign prostate hyperplasia patients with PSA $>2.5 \mathrm{ng} / \mathrm{ml}$ (AUCs of 83.7 and $91.8 \%$, respectively) (40).
Serum miR-191 expression was found to be 5.3-fold higher in renal cell carcinoma patients than that in controls (41). Plasma miR-33b was significantly lower in bladder cancer cases (28) than that in the controls, whereas the serum expression of miR-33b was enhanced in the UTUC cases in the present study. This disparity should be cautiously interpreted according to the possibly different cellular and molecular mechanisms underlying UTUC and lower tract urothelial carcinoma, even if the pathogenetic similarity of UTUC and bladder cancer has been previously reported $(4,42)$. These miRNAs may serve as a 'fingerprint' to identify the presence of urinary cancers. Thus, the diagnostic capacity of these four circulating miRNAs (miR-16, miR-26a, miR-33b and miR-191) in the precise detection of UTUC may be impaired by their involvement in other urinary cancers. Further studies should be performed to confirm the functions of these four circulating miRNAs in urinary cancers.

In the present study, UTUC was found to be associated with serum miR-423 and miR-664. Circulating miR-423 may be useful as a stable blood-based biomarker for numerous diseases, including heart failure (43) and acute graft-vs-host disease (44). However, few studies have demonstrated the implication of serum miR-423 in cancers. Previous studies have shown that miR-423 is increased in head and neck squamous cell carcinomas (45), and is highly expressed in infiltrating ductal carcinomas in women, who later developed metastasis (46). These findings suggested that miR-423 has 
a function in cancer development. To the best of our knowledge, no previous study has revealed the association between circulating miR-664 and disease prediction. However, the upregulation of miR-664 contributed to enhanced tumorigenesis in a hepatocellular carcinoma cell line (47), suggesting its potential oncogenic behavior. The lack of results showing the association between cancer and circulating miR-664 and miR-423 levels indicates marked cancer specificity of these two miRNAs in UTUC diagnosis. Of note, the high diagnostic capacity (AUC $=99.8 \%$ ) of serum miR-664 in detecting UTUC may be applied in future studies.

Other molecular markers have also been investigated in samples from UTUC patients using immunohistochemistry or in situ hybridization. E-cadherin and hypoxia-inducible factor- $1 \alpha$ are prognostic factors in UTUC, and the expression of human telomerase RNA is a useful indicator for the diagnosis and prognosis of UTUC (48-50). However, these molecular markers are more associated with the prognosis rather than the diagnosis of UTUC, and depend on conventional detection methods. Compared with circulating miRNA detection, the conventional diagnostic methods for UTUC, including ureteroscopy, urine cytology, excretory urography and ultrasonography, cause discomfort among patients and are time-consuming and invasive. Such methods also lack sensitivity and specificity, which may delay diagnosis and treatment. By contrast, the circulating miRNAs identified in the present study could allow for early diagnosis with high sensitivity and specificity.

However, the results of the present study need to be interpreted with caution, as the diagnosis as UTUC has several limitations. First, as UTUC is an uncommon disease, the sample size was relatively small, which may have limited the diagnostic capacity of serum miRNA. Second, the miRNA expression levels were higher in the case group than those in the control group, even if the initial sequencing stage showed that the levels of several miRNAs were downregulated in the case group. The discrepancy between the results of the two stages may be attributed to the relatively low abundance of reduced miRNAs, which was beyond the threshold of RT-qPCR detection. Third, the results of the present study were based on a retrospective study. Thus, a future prospective cohort is required to validate the results of the present study. Finally, other factors, including race and methodology, possibly affected the final results.

In conclusion, a 10 serum miRNA-based expression profile that was able to accurately discern UTUC individuals from the controls was identified. Although the manner by which UTUC-specific miRNAs enter the circulation is unknown, the identified serum miRNAs are useful biomarkers for UTUC detection. Such serum-based assays may result in improved screening compared with stool-based or endoscopic screening methods. Circular miRNA-based screening for UTUC must be tested on large cohorts to confirm and increase their sensitivity and specificity.

\section{Acknowledgements}

This work was supported by the Program for Development of Innovative Research Team of the First Affiliated Hospital of Nanjing Medical University, the Provincial Initiative Program for Excellency Disciplines of Jiangsu Province, the National Natural Science Foundation of China (grant nos. 81272832 and
81201997), the Natural Science Foundation of Jiangsu Province (grant no. BK2011848), the Six Major Talent Peak Project of Jiangsu Province (grant no. 2011-WS-121), the Priority Academic Program Development of Jiangsu Higher Education Institutions (PAPD) and Jiangsu Provincial Special Program of Medical Science (grant no. BL2012027). The funders had no role in study design, data collection and analysis, decision to publish, or preparation of the manuscript.

\section{References}

1. Hall MC, Womack S, Sagalowsky AI, Carmody T, Erickstad MD and Roehrborn CG: Prognostic factors, recurrence and survival in transitional cell carcinoma of the upper urinary tract: A 30-year experience in 252 patients. Urology 52: 594-601, 1998.

2. Munoz JJ and Ellison LM: Upper tract urothelial neoplasms: Incidence and survival during the last 2 decades. J Urol 164: 1523-1525, 2000.

3. Rouprêt M, Zigeuner R, Palou J, Boehle A, Kaasinen E, Sylvester R, Babjuk M and Oosterlinck W: European guidelines for the diagnosis and management of upper urinary tract urothelial cell carcinomas: 2011 update. Eur Urol 59: 584-594, 2011.

4. Krabbe LM, Lotan Y, Bagrodia A, Gayed BA, Darwish OM, Youssef RF, Bolenz C, Sagalowsky AI, Raj GV, Shariat SF, et al: Prospective comparison of molecular signatures in urothelial cancer of the bladder and the upper urinary tract-is there evidence for discordant biology? J Urol 191: 926-931, 2014.

5. Hautmann RE, de Petriconi RC, Pfeiffer C and Volkmer BG: Radical cystectomy for urothelial carcinoma of the bladder without neoadjuvant or adjuvant therapy: Long-term results in 1100 patients. Eur Urol 61: 1039-1047, 2012.

6. Margulis V, Shariat SF, Matin SF, Kamat AM, Zigeuner R, Kikuchi E, Lotan Y, Weizer A, Raman JD and Wood CG; The Upper Tract Urothelial Carcinoma Collaboration: Outcomes of radical nephroureterectomy: A series from the upper tract urothelial carcinoma collaboration. Cancer 115: 1224-1233, 2009.

7. Winter J, Jung S, Keller S, Gregory RI and Diederichs S: Many roads to maturity: microRNA biogenesis pathways and their regulation. Nat Cell Biol 11: 228-234, 2009.

8. Lin S and Gregory RI: MicroRNA biogenesis pathways in cancer. Nat Rev Cancer 15: 321-333, 2015.

9. Visone R and Croce CM: MiRNAs and cancer. Am J Pathol 174: 1131-1138, 2009.

10. Croce CM: Causes and consequences of microRNA dysregulation in cancer. Nat Rev Genet 10: 704-714, 2009.

11. Mitchell PS, Parkin RK, Kroh EM, Fritz BR, Wyman SK, Pogosova-Agadjanyan EL, Peterson A, Noteboom J, O'Briant KC, Allen A, et al: Circulating microRNAs as stable blood-based markers for cancer detection. Proc Natl Acad Sci USA 105: 10513-10518, 2008.

12. Chen X, Ba Y, Ma L, Cai X, Yin Y, Wang K, Guo J, Zhang Y, Chen J, Guo X, et al: Characterization of microRNAs in serum: A novel class of biomarkers for diagnosis of cancer and other diseases. Cell Res 18: 997-1006, 2008.

13. Huang X, Liang M, Dittmar R and Wang L: Extracellular microRNAs in urologic malignancies: Chances and challenges. Int J Mol Sci 14: 14785-14799, 2013.

14. Izquierdo L, Ingelmo-Torres M, Mallofré C, Lozano JJ, Verhasselt-Crinquette M, Leroy X, Colin P, Comperat E, Roupret M, Alcaraz A and Mengual L: Prognostic value of microRNA expression pattern in upper tract urothelial carcinoma. BJU Int 113: 813-821, 2014.

15. Liu R, Zhang C, Hu Z, Li G, Wang C, Yang C, Huang D, Chen X, Zhang $\mathrm{H}$, Zhuang R, et al: A five-microRNA signature identified from genome-wide serum microRNA expression profiling serves as a fingerprint for gastric cancer diagnosis. Eur J Cancer 47: 784-791, 2011.

16. Yu S, Liu Y, Wang J, Guo Z, Zhang Q, Yu F, Zhang Y, Huang K, Li Y, Song E, et al: Circulating microRNA profiles as potential biomarkers for diagnosis of papillary thyroid carcinoma. J Clin Endocrinol Metab 97: 2084-2092, 2012.

17. Li LM, Hu ZB, Zhou ZX, Chen X, Liu FY, Zhang JF, Shen HB, Zhang CY and Zen K: Serum microRNA profiles serve as novel biomarkers for HBV infection and diagnosis of HBV-positive hepatocarcinoma. Cancer Res 70: 9798-9807, 2010. 
18. Yang C, Wang C, Chen X, Chen S, Zhang Y, Zhi F, Wang J, Li L, Zhou X, Li N, et al: Identification of seven serum microRNAs from a genome-wide serum microRNA expression profile as potential noninvasive biomarkers for malignant astrocytomas. Int J Cancer 132: 116-127, 2013.

19. Sauter G, Algaba F, Amin M, et al: Tumours of the urinary system: Non-invasive urothelial neoplasia. In Eble JN, Sauter G, Epstein JI and Sesterhenn IA (eds.): The WHO Classification of Tumours of the Urinary System and Male Genital Organs. IARC Press, Lyon, pp110-114, 2004.

20. Sobin DH and Witteking CH (eds.): TNM Classification of Malignant Tumours. 6th edition. Wiley-Liss, New York, NY, pp 199-202, 2002.

21. Sanders I, Holdenrieder S, Walgenbach-Brunagel G, Walgenbach-Brünagel $G$, von Ruecker A, Kristiansen G, Müller SC and Ellinger J: Evaluation of reference genes for the analysis of serum miRNA in patients with prostate cancer, bladder cancer and renal cell carcinoma. Int J Urol 19: 1017-1025, 2012.

22. Cortez MA, Bueso-Ramos C, Ferdin J, Lopez-Berestein G, Sood AK and Calin GA: MicroRNAs in body fluids-the mix of hormones and biomarkers. Nat Rev Clin Oncol 8: 467-477, 2011

23. Zen K and Zhang CY: Circulating microRNAs: A novel class of biomarkers to diagnose and monitor human cancers. Med Res Rev 32: 326-348, 2012.

24. Brosnan JA and Iacobuzio-Donahue CA: A new branch on the tree: Next-generation sequencing in the study of cancer evolution. Semin Cell Dev Biol 23: 237-242, 2012

25. Pritchard CC, Cheng HH and Tewari M: MicroRNA profiling: Approaches and considerations. Nat Rev Genet 13: 358-369, 2012.

26. Mlcochova H, Hezova R, Stanik M and Slaby O: Urine microRNAs as potential noninvasive biomarkers in urologic cancers. Urol Oncol 32: 41 e41-e49, 2014.

27. Mengual L, Lozano JJ, Ingelmo-Torres M, Gazquez C, Ribal MJ and Alcaraz A: Using microRNA profiling in urine samples to develop a non-invasive test for bladder cancer. Int J Cancer 133 2631-2641, 2013

28. Adam L, Wszolek MF, Liu CG, Jing W, Diao L, Zien A, Zhang JD, Jackson D and Dinney CP: Plasma microRNA profiles for bladder cancer detection. Urol Oncol 31: 1701-1708, 2013

29. Cookson VJ, Bentley MA, Hogan BV, Horgan K, Hayward BE, Hazelwood LD and Hughes TA: Circulating microRNA profiles reflect the presence of breast tumours but not the profiles of microRNAs within the tumours. Cell Oncol (Dordr) 35: 301-308, 2012.

30. Chung YW, Bae HS, Song JY, Lee JK, Lee NW, Kim T and Lee KW: Detection of microRNA as novel biomarkers of epithelial ovarian cancer from the serum of ovarian cancer patients. Int J Gynecol Cancer 23: 673-679, 2013.

31. Tanaka K, Miyata H, Yamasaki M, Sugimura K, Takahashi T, Kurokawa Y, Nakajima K, Takiguchi S, Mori M and Doki Y: Circulating miR-200c levels significantly predict response to chemotherapy and prognosis of patients undergoing neoadjuvant chemotherapy for esophageal cancer. Ann Surg Oncol 20 (Suppl 3): S607-S615, 2013

32. Wang HY, Yan LX, Shao Q, Fu S, Zhang ZC, Ye W, Zeng YX and Shao JY: Profiling plasma microRNA in nasopharyngeal carcinoma with deep sequencing. Clin Chem 60: 773-782, 2014.

33. Kannan M, Mohan KV, Kulkarni S and Atreya C: Membrane array-based differential profiling of platelets during storage for 52 miRNAs associated with apoptosis. Transfusion 49: 1443-1450, 2009

34. Zhang C, Wang C, Chen X, Yang C, Li K, Wang J, Dai J, Hu Z, Zhou X, Chen L, et al: Expression profile of microRNAs in serum: A fingerprint for esophageal squamous cell carcinoma. Clin Chem 56: 1871-1879, 2010.
35. Lionetti M, Musto P, Di Martino MT, Fabris S, Agnelli L, Todoerti K, Tuana G, Mosca L, Gallo Cantafio ME, Grieco V, et al: Biological and clinical relevance of miRNA expression signatures in primary plasma cell leukemia. Clin Cancer Res 19: 3130-3142, 2013.

36. Kanaan Z, Roberts H, Eichenberger MR, Billeter A, Ocheretner G, Pan J, Rai SN, Jorden J, Williford A and Galandiuk S: A plasma microRNA panel for detection of colorectal adenomas: A step toward more precise screening for colorectal cancer. Ann Surg 258: 400-408, 2013 .

37. Zheng G, Wang H, Zhang X, Yang Y, Wang L, Du L, Li W, Li J, Qu A, Liu Y and Wang C: Identification and validation of reference genes for qPCR detection of serum microRNAs in colorectal adenocarcinoma patients. PLoS One 8: e83025, 2013.

38. Wang Y, Gu J, Roth JA, Hildebrandt MA, Lippman SM, Ye Y, Minna JD and Wu X: Pathway-based serum microRNA profiling and survival in patients with advanced stage non-small cell lung cancer. Cancer Res 73: 4801-4809, 2013.

39. Liu C, Iqbal J, Teruya-Feldstein J, Shen Y, Dabrowska MJ, Dybkaer K, Lim MS, Piva R, Barreca A, Pellegrino E, et al: MicroRNA expression profiling identifies molecular signatures associated with anaplastic large cell lymphoma. Blood 122: 2083-2092, 2013

40. Mahn R, Heukamp LC, Rogenhofer S, von Ruecker A, Müller SC and Ellinger J: Circulating microRNAs (miRNA) in serum of patients with prostate cancer. Urology 77: 1265, e9-e16, 2011.

41. Hauser S, Wulfken LM, Holdenrieder S, Moritz R, Ohlmann CH, Jung V, Becker F, Herrmann E, Walgenbach-Brünagel G, von Ruecker A, et al: Analysis of serum microRNAs (miR-26a-2*, miR-191, miR-337-3p and miR-378) as potential biomarkers in renal cell carcinoma. Cancer Epidemiol 36: 391-394, 2012.

42. Zhang Z,Furge KA, Yang XJ, Teh BT and Hansel DE: Comparative gene expression profiling analysis of urothelial carcinoma of the renal pelvis and bladder. BMC Med Genomics 3: 58, 2010.

43. Tijsen AJ, Creemers EE, Moerland PD, de Windt LJ, van der Wal AC, Kok WE and Pinto YM: MiR423-5p as a circulating biomarker for heart failure. Circ Res 106: 1035-1039, 2010.

44. Xiao B, Wang Y, Li W, Baker M, Guo J, Corbet K, Tsalik EL, Li QJ, Palmer SM, Woods CW, et al: Plasma microRNA signature as a noninvasive biomarker for acute graft-versus-host disease. Blood 122: 3365-3375, 2013

45. Hui AB, Lenarduzzi M, Krushel T, Waldron L, Pintilie M, Shi W, Perez-Ordonez B, Jurisica I, O'Sullivan B, Waldron J, et al: Comprehensive MicroRNA profiling for head and neck squamous cell carcinomas. Clin Cancer Res 16: 1129-1139, 2010

46. Farazi TA, Horlings HM, Ten Hoeve JJ, Mihailovic A, Halfwerk H, Morozov P, Brown M, Hafner M, Reyal F, van Kouwenhove M, et al: MicroRNA sequence and expression analysis in breast tumors by deep sequencing. Cancer Res 71: 4443-4453, 2011

47. Yang H, Cho ME, Li TW, Peng H, Ko KS, Mato JM and Lu SC: MicroRNAs regulate methionine adenosyltransferase $1 \mathrm{~A}$ expression in hepatocellular carcinoma. J Clin Invest 123: 285-298, 2013

48. Fromont G, Rouprêt M, Amira N, Sibony M, Vallancien G, Validire $\mathrm{P}$ and Cussenot $\mathrm{O}$ : Tissue microarray analysis of the prognostic value of E-cadherin, Ki67, p53, p27, survivin and MSH2 expression in upper urinary tract transitional cell carcinoma. Eur Urol 48: 764-770, 2005

49. Nakanishi K, Kawai T, Hiroi S, Kumaki F, Torikata C, Aurues T and Ikeda T: Expression of telomerase mRNA component (hTR) in transitional cell carcinoma of the upper urinary tract. Cancer 86: 2109-2116, 1999.

50. Nakanishi K, Hiroi S, Tominaga S, Aida S, Kasamatsu H, Matsuyama S, Matsuyama T and Kawai T: Expression of hypoxia-inducible factor-1alpha protein predicts survival in patients with transitional cell carcinoma of the upper urinary tract. Clin Cancer Res 11: 2583-2590, 2005. 